chend internationaler Standards müssen Schutz und Sicherheit oberstes Ziel von Täterarbeit und die Unterstützung jedes einzelnen Opfers Bestandteil des Programms sein. ${ }^{36}$ In Deutschland und auch in Österreich existieren derzeit leider noch viele Programme, die diese Standards nicht erfüllen und die Sicherheit der Opfer nicht gewährleisten. Diese Programme sollten keine öffentliche Finanzierung erhalten. Fraueneinrichtungen sollen in Zukunft in der Täterarbeit eine federführende Rolle einnehmen und ihre Angebote für Frauen, die sich entscheiden, mit dem gewalttätigen Partner weiter zusammenzuleben, erweitern.

\section{Solidarität und europäische Vernetzung}

Solidarität und gegenseitige Unterstützung auf europäischer Ebene sind wichtig, um das Ziel adäquater Hilfsangebote für Frauen und ihre Kinder zu erreichen. Dahin ist es noch ein weiter Weg, und insbesondere unsere „Schwestern“ in Osteu- ropa benötigen unsere Solidarität und auch Unterstützung beim Aufbau und Erhalt ihrer Einrichtungen, da sie wenig bis keine Mittel vom Staat erhalten. Alles was wir dazu beitragen können - z. B. Mitwirkung am WAVE Netzwerk, Spenden an das SAVE Konto von WAVE ${ }^{37}$, von StädtepartnerInnenschaften, gemeinsame Projekte, Unterstützung beim Fundraising usw. - stärkt das Recht von Frauen und Kindern auf ein Leben frei von Gewalt in Europa. Die deutschen Fraueneinrichtungen sind wichtig für die Vernetzung und haben viel zu bieten, ich möchte daher alle herzlich ermutigen, einen Teil der Ressourcen für das Engagement auf europäischer Ebene einzusetzen. Damit verbessern wir auch grenzübergreifende Kooperationen und die Hilfen für Frauen, die in mehr als einem Land von Gewalt betroffen sind.

36 Council of Europe 2008 Siehe www.wave-network.org. 37 Siehe www.wave-network.org.

\title{
Integrationsprogramm für von Gewalt betroffene Frauen
}

Ein auf Vorschlag des spanischen Ministers für Arbeit und Migration, Celestino Corbacho, und der Ministerin für Gleichstellung, Bibiana Aido, im November 2008 verabschiedetes Programm bietet Frauen, die von geschlechtsspezifischer Gewalt betroffen sind, Hilfen zur sozialen Integration und Unterstützung bei der Aufnahme einer Beschäftigung. Parallel erhalten Unternehmen, die Gewaltopfer einstellen, eine Gutschrift der Sozialversicherungsbeiträge in Höhe von bis zu 1.500 Euro pro Jahr. Die Arbeitsaufnahme soll durch ein Ausund Weiterbildungsangebot erleichtert werden, das einen besonderen Fokus auf die Stärkung des Selbstwertgefühls, das Empowerment und damit die Motivation legt. Unterstützung wird ebenfalls beim Umzug in eine andere Region, der Einrichtung einer neuen Wohnung sowie der Kinderbetreuung oder Versorgung pflegebedürftiger Angehörigen geleistet, falls dadurch eine neue Beschäftigung angetreten werden kann. Auch für einen Start in die Selbständigkeit werden Hilfen angeboten.

Vorausgesetzt wird der Nachweis erlebter geschlechtsspezifischer Gewalt, der u.a. durch Vorlage entsprechender Schutzbeschlüsse erbracht werden kann.
Andererseits wird durch datenschutzrechtliche Regelungen ein Mechanismus vorgesehen, durch welchen die Privatsphäre der Gewaltbetroffenen - z.B. im Betrieb wie auch auf dem Wohnungsmarkt - gewahrt werden soll. Dies auch im Hinblick auf die Vorbeugung erneuter Viktimisierung durch Bloßstellung und Ausgrenzung.

Für die Umsetzung des Programms stehen jährlich 11,1 Millionen Euro zur Verfügung. Es wird davon ausgegangen, dass bis zu 8.000 Frauen von den spezifischen und individuell ausgerichteten Hilfen profitieren können.

Das Integrationsprogramm beruht in erster Linie auf dem spanischen Gender Violence Act aus 2004 und dient der Umsetzung des Gleichberechtigungsrechts wie mehrerer Normen der Sozialgesetzgebung.

Zusammengestellt von Nancy Gage-Lindner, Mitglied der Kommission Gewalt gegen Frauen und Kinder des djb. Deutschsprachige Quelle: Aktuelle Nachrichten aus Spanien, Ausgabe 16.-31.3.2009, Botschaft von Spanien, Abt. für Arbeit und Migration/Berlin; vollständiger Regelungstext nebst Begründung: http://www.boe.es/boe/dias/2008/12/10/pdfs/A4936749373.pdf.

Bald nach Verabschiedung des deutschen Gewaltschutzgesetzes gab das Bundesministerium der Justiz eine wissenschaftliche Evaluation der Gerichtspraxis in Auftrag. Die Bund-Länder-AG häusliche Gewalt wertete diese Studie aus und legte ihre Schlussfolgerungen für Bund und Länder im Frühjahr 2009 vor. Gleichzeitig befasst sich die Bund-Länder-AG Häusliche Gewalt mit der rechtspolitischen Entwicklung in Spanien mit dem Ziel, hieraus für Deutschland weitere Erkenntnisse zu gewinnen. Im diesem Kontext stellt der djb eine Auswahlbibliographie der Literatur zur rechtspolitischen Entwicklung in Spanien bereit. Diese ist als Download abrufbar unter www.djb.de/kommssionen/kommission-gewalt-gegen-frauen-und-kinder/. 